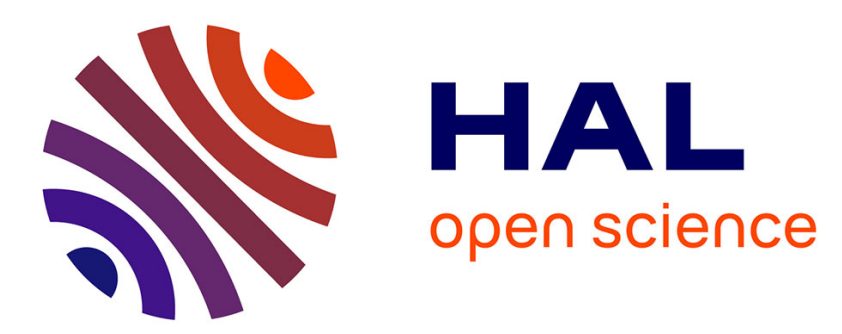

\title{
Edge detection using topological gradients: a scale-space approach
}

\author{
Samuel Amstutz, Jérôme Fehrenbach
}

\section{To cite this version:}

Samuel Amstutz, Jérôme Fehrenbach. Edge detection using topological gradients: a scale-space approach. Journal of Mathematical Imaging and Vision, 2015, 10.1007/s10851-015-0558-z . hal01025706

\section{HAL Id: hal-01025706 \\ https://hal.science/hal-01025706}

Submitted on 18 Jul 2014

HAL is a multi-disciplinary open access archive for the deposit and dissemination of scientific research documents, whether they are published or not. The documents may come from teaching and research institutions in France or abroad, or from public or private research centers.
L'archive ouverte pluridisciplinaire HAL, est destinée au dépôt et à la diffusion de documents scientifiques de niveau recherche, publiés ou non, émanant des établissements d'enseignement et de recherche français ou étrangers, des laboratoires publics ou privés. 


\title{
Edge detection using topological gradients: a scale-space approach
}

\author{
Samuel Amstutz · Jérôme Fehrenbach
}

Received: date / Accepted: date

\begin{abstract}
We provide in this paper a link between two methods of edge detection: edge detection using scale-space analysis, and edge detection using topological asymptotic analysis. More precisely, we show that the topological gradient associated with an image $u$ is given by a combination of the gradients of two smoothed versions of the image $u$ at two different scales, namely $\varphi \star u$ and $(\varphi \star \varphi) \star u$, where $\varphi$ is the fundamental solution of the elliptic restoration equation. In the same setting we propose a new edge detector based on the maximization of the variance of the image. Then we generalize our approach to Gaussian kernels considering a topological asymptotic analysis of the parabolic heat equation. A numerical comparison of these detectors together with the Canny edge detector is presented.
\end{abstract}

Keywords Edge detection - Topological gradient . Heat equation · Scale space

\section{Introduction}

Edge detection using topological asymptotic analysis was introduced in [7] and applied to image restoration. It lead to numerous variants and applications such as inpainting [5], color image restoration [6], super-resolution and demosaicking [12]. This approach provides a non linear edge detector that is based on the elliptic restoration equation (1) below. Topological asymptotic anal-

\section{S. Amstutz}

Laboratoire de Mathématiques d'Avignon, Université d'Avignon, France

E-mail: samuel.amstutz@univ-avignon.fr

\section{J. Fehrenbach}

Institut de Mathématiques de Toulouse, CNRS and Université Paul Sabatier, Toulouse, France

E-mail: jerome.fehrenbach@math.univ-toulouse.fr ysis provides the asymptotic variation of a cost function associated with the solution of a partial differential equation when the domain is modified by an infinitesimal topology perturbation $[15,20,21,14]$. When an insulating edge segment is inserted, the asymptotic variation of the cost function was first derived in [1] for the Laplace equation and extended to the restoration equation (1) in [7].

Given an image $f$ defined on a rectangular domain $\Omega$, let $u_{0}$ be the solution of the elliptic restoration equation:

$\begin{cases}-\operatorname{div}(c \nabla u)+u=f & \text { in } \Omega, \\ \nabla u . n=0 & \text { on } \partial \Omega .\end{cases}$

The solution of (1) provides a regularized version $u_{0}$ of the image $f$ that is the minimizer of

$j(u)=\frac{1}{2} \int_{\Omega}|u-f|^{2}+\frac{1}{2} \int_{\Omega} c|\nabla u|^{2}$.

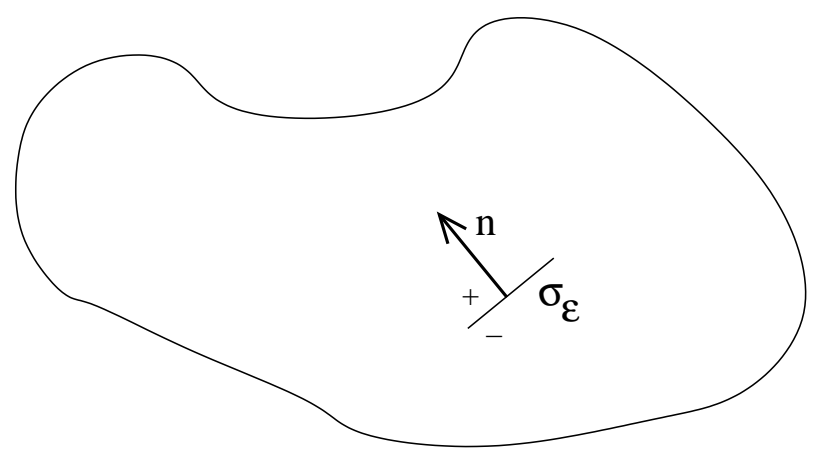

Fig. 1 The domain $\Omega$, the crack $\sigma_{\epsilon}$ and the normal vector $\mathbf{n}$. The jump convention is the following: $[v]=v^{+}-v^{-}$.

The perturbed domain $\Omega_{\epsilon}$ is obtained by the insertion at some point $x_{0}$ of an insulating crack $\sigma_{\epsilon}$ of length 
$2 \epsilon$, see Figure 1 . The perturbed solution $u_{\epsilon}$ is the solution of the perturbed equation:

$$
\left\{\begin{array}{lr}
-\operatorname{div}\left(c \nabla u_{\epsilon}\right)+u_{\epsilon}=f & \text { in } \Omega_{\epsilon}, \\
\nabla u_{\epsilon} \cdot n=0 & \text { on } \partial \Omega, \\
\nabla u_{\epsilon} \cdot n=0 & \text { on } \sigma_{\epsilon} .
\end{array}\right.
$$

The italic letter $n$ stands for the normal to the boundary of the domain $\Omega$. The bold letter $\mathbf{n}$ will denote the normal vector to the crack.

The main result in [7] is the following: the variation of the cost function

$$
j_{1}(\epsilon)=J_{1, \epsilon}\left(u_{\epsilon}\right)=\int_{\Omega_{\epsilon}}\left|\nabla u_{\epsilon}\right|^{2}
$$

when an insulating crack of length $2 \epsilon$ is inserted at the point $x_{0}$ with a normal vector $\mathbf{n}$ is equivalent when $\epsilon \rightarrow 0$ to $g_{1}\left(x_{0}, \mathbf{n}\right) \epsilon^{2}$ with

$g_{1}\left(x_{0}, \mathbf{n}\right)=-c \pi\left(\nabla u_{0}\left(x_{0}\right) \cdot \mathbf{n}\right)\left(\nabla p\left(x_{0}\right) \cdot \mathbf{n}\right)-\pi\left|\nabla u_{0}\left(x_{0}\right) \cdot \mathbf{n}\right|^{2}$,

where the adjoint state $p$ solves

$\begin{cases}-\operatorname{div}(c \nabla p)+p=2 \Delta u_{0} & \text { in } \Omega, \\ \nabla p \cdot n=0 & \text { on } \partial \Omega .\end{cases}$

Alternatively, we have $g_{1}\left(x_{0}, \mathbf{n}\right)=-\mathbf{n}^{T} M_{1}\left(x_{0}\right) \mathbf{n}$ with the matrix

$$
\begin{aligned}
M_{1}\left(x_{0}\right)= & \frac{1}{2} c \pi\left(\nabla u_{0}\left(x_{0}\right) \nabla p\left(x_{0}\right)^{T}+\nabla p\left(x_{0}\right) \nabla u_{0}\left(x_{0}\right)^{T}\right) \\
& +\pi \nabla u_{0}\left(x_{0}\right) \nabla u_{0}\left(x_{0}\right)^{T} .
\end{aligned}
$$

Therefore the optimal direction of the normal of the crack at the point $x_{0}$ (the direction where the cost function $j_{1}$ decreases the most) is the eigenvector associated with the largest eigenvalue of $M_{1}$, and the contours are likely to be located at the points where this largest eigenvalue is maximal. The largest eigenvalue of the matrix $M_{1}$ is then an edge detector that we denote $g_{1}\left(x_{0}\right)$.

When a segment is inserted in an image, the process can be iterated and a union of disjoint segments can be added and form a curve, whose length is the sum of the lengths of the segments. A series of works based on the refinement of this simple idea was initiated in [17]. When an insulating curve $K$ is inserted in a domain and the restoration equation is applied then the solution is the minimizer of

$$
J_{K}(u)=\frac{1}{2} \int_{\Omega}|u-f|^{2}+\frac{1}{2} \int_{\Omega \backslash K} c|\nabla u|^{2} .
$$

Among the curves of given length, this is exactly the minimizer of Mumford-Shah functional [16]:

$$
J_{K}(u)=\frac{1}{2} \int_{\Omega}|u-f|^{2}+\frac{1}{2} \int_{\Omega \backslash K} c|\nabla u|^{2}+\beta \mathcal{H}^{1}(K),
$$

Table 1 Polarization tensor for different inclusions. The conductivity of the background is $c$, the normal of the rectangle/crack is $\mathbf{n}$ and the tangent is $\mathbf{t}$.)

\begin{tabular}{ll}
\hline Inclusion & Polarization tensor \\
\hline Insulating disk & $\pi c I d$ \\
Disk w. conductivity $\kappa c$ & $\pi c \frac{1-\kappa}{1+\kappa} I d$ \\
Rectangle w. conductivity $\kappa c$ & $2 c(1-\kappa)\left(\frac{1}{\kappa} \mathbf{n n}^{T}+\mathbf{t t}^{T}\right)$ \\
Insulating crack & $\pi c \mathbf{n} \mathbf{n}^{T}$ \\
\hline
\end{tabular}

where $\mathcal{H}^{1}(K)$ is the one-dimensional Hausdorff measure of the set $K$. This connection between the insertion of segments and the Mumford-Shah functional is rigourously detailed in [10]. A family of functionals $J_{\epsilon, \kappa}$ is defined, and if $\epsilon \rightarrow 0$ and simultaneously $\kappa=\kappa(\epsilon)=o(\epsilon)$ then the functional $J_{\epsilon, \kappa(\epsilon)} \Gamma$-converges to Mumford-Shah functional. The perturbation of the domain considered in $[17,10]$ is a circular inclusion with an almost insulating material of conductivity $\kappa c$, which is not the same as the perfectly insulating crack considered in [7]. We also mention [8] where the case of a rectangular inclusion with sides $\epsilon \times \epsilon^{2}$ and conductivity $\kappa c$ with $0<\kappa<1$ is addressed. The approach of $[17$, 10] provides a self-adjoint problem, and the topological gradient related to the insertion of a disk/crack is

$g_{2}\left(x_{0}, \mathbf{n}\right)=-\nabla u_{0}^{T}\left(x_{0}\right) \mathcal{P} \nabla u_{0}\left(x_{0}\right)$,

where $\mathcal{P}$ is the polarization tensor of the inclusion. We summarize in Table 1 examples of polarization tensors for different inclusions. In order to present a unified approach, we shall in this work adapt the results of $[17$, 10] by replacing the circular/rectangular inclusion with different conductivity by a perfectly insulating crack.

We review in detail these results in section 2 . We also define a new cost function $j_{3}(\epsilon)$ associated with the perturbed problem (3), which amounts to maximizing the variance of the perturbed image. The associated topological gradient is denoted by $g_{3}$.

Considering a crack with optimal orientation the edge detector $g_{2}$ is exactly the squared norm of the gradient of the image $u_{0}$ which is a smoothed version of the original image $f$. We provide in section 3 a link between the edge detectors $g_{1}$ and $g_{3}$ on one hand, and a scale-space approach $[22,11,13,18]$ on the other hand. Specifically, the topological gradients $g_{1}$ and $g_{3}$ can be identified as combinations of the gradients of the image $f$ smoothed at two different scales. The smoothing kernel is the fundamental solution $\varphi$ of the elliptic restoration equation (1). It is given by a modified Bessel function which presents a singularity at the origin.

Standard scale-space theory uses Gaussian kernels, which are smooth at the origin in contrast to the kernel 
$\varphi$. In order to emphasize the link with scale-space representations and to interpret the results obtained with smooth kernels, we extend in section 4 the approach presented above to the parabolic restoration equation

$$
\left\{\begin{array}{l}
\partial_{t} u=\Delta u \quad \text { in } \Omega \times[0, T] \\
\nabla u \cdot n=0 \quad \text { on } \partial \Omega \times[0, T] \\
u(t=0)=f .
\end{array}\right.
$$

This is natural since the fundamental solution of the parabolic restoration equation (7) is a Gaussian with standard deviation $\sqrt{2 t}$. Different cost functions associated with the perturbation of equation (7) by an infinitesimal crack are presented in Section 4. This extension allows to define new edge detectors $h_{1}, h_{3}, h_{4}$. The Canny edge detector denoted $h_{2}$, as a sort of parabolic counterpart of $g_{2}$, will serve as a reference.

All the theoretical results are illustrated in Section 5 , where the different edge detectors $g_{1}, g_{2}, g_{3}$ are presented as well as the detectors $h_{1}, h_{2}, h_{3}, h_{4}$ derived from parabolic restoration. We also provide a quantitative comparison of these detectors based on the evaluation of the noise level reduction and of the spatial resolution.

\section{Topological asymptotics for elliptic restoration}

In this section we review known results for the perturbation of the restoration equation by an insulating crack, and propose a new cost function that allows to design a new edge indicator.

We consider a bounded domain $\Omega$, and a line segment $\sigma_{\epsilon}$ of length $2 \epsilon$ located at the point $x_{0} \in \Omega$ with unit normal vector $\mathbf{n}$. The elliptic restoration problem is equation (1) and the perturbed problem is equation (3). For well-posedness we assume that $f \in L^{2}(\Omega)$. We present here topological asymptotics results in a particular case appropriate to edge detection. For a more general framework the reader is referred to $[1,2]$.

The variational formulation of (1) reads

$$
\left\{\begin{array}{l}
\text { Find } u \in H^{1}(\Omega) \text { s.t. } \\
\forall v \in H^{1}(\Omega), a(u, v)=\ell(v),
\end{array}\right.
$$

where

$$
a(u, v)=\int_{\Omega} c \nabla u \cdot \nabla v+u v \text { and } \ell(v)=\int_{\Omega} f v .
$$

The variational formulation of the perturbed problem (3) reads

$$
\left\{\begin{array}{l}
\text { Find } u \in H^{1}\left(\Omega_{\epsilon}\right) \text { s.t. } \\
\forall v \in H^{1}\left(\Omega_{\epsilon}\right), a_{\epsilon}(u, v)=\ell(v)
\end{array}\right.
$$

where

$$
a_{\epsilon}(u, v)=\int_{\Omega_{\epsilon}} c \nabla u . \nabla v+u v
$$

We consider a cost function $j(\epsilon)=J\left(u_{\epsilon}\right)$ that satisfies the following hypotheses:

(H1) $J\left(u_{\epsilon}\right)-J\left(u_{0}\right)=L\left(u_{\epsilon}-u_{0}\right)+\epsilon^{2} \delta J+o\left(\epsilon^{2}\right)$, with $L \in \mathcal{L}\left(H^{1}(\Omega), \mathbb{R}\right)$ and $\delta J \in \mathbb{R}$,

(H2) $\left(a_{\epsilon}-a_{0}\right)\left(u_{0}, p_{\epsilon}\right)=\epsilon^{2} \delta a+o\left(\epsilon^{2}\right)$, where the adjoint state $p_{\epsilon}$ solves: $p_{\epsilon} \in H^{1}\left(\Omega_{\epsilon}\right)$ and

$$
\forall v \in H^{1}\left(\Omega_{\epsilon}\right), a_{\epsilon}\left(v, p_{\epsilon}\right)=-L v
$$

Theorem 1 Under these hypotheses, the variation of the cost function $j(\epsilon)$ when $\epsilon \rightarrow 0$ is given by the following asymptotic expansion:

$$
j(\epsilon)=j(0)+\epsilon^{2}(\delta a+\delta J)+o\left(\epsilon^{2}\right) .
$$

Proof This calculation is standard [3]:

$$
\begin{aligned}
j(\epsilon)-j(0) & =J\left(u_{\epsilon}\right)-J\left(u_{0}\right) \\
& =L\left(u_{\epsilon}-u_{0}\right)+\epsilon^{2} \delta J+o\left(\epsilon^{2}\right) \\
& =-a_{\epsilon}\left(u_{\epsilon}-u_{0}, p_{\epsilon}\right)+\epsilon^{2} \delta J+o\left(\epsilon^{2}\right) \\
& =\left(a_{\epsilon}-a_{0}\right)\left(u_{0}, p_{\epsilon}\right)+\epsilon^{2} \delta J+o\left(\epsilon^{2}\right) \\
& =\epsilon^{2}(\delta a+\delta J)+o\left(\epsilon^{2}\right) .
\end{aligned}
$$

Theorem 2 Under the hypotheses above it holds

$$
\delta a=-\nabla u_{0}\left(x_{0}\right)^{T} \mathcal{P} \nabla p_{0}\left(x_{0}\right),
$$

where the polarization tensor is given by $\mathcal{P}=c \pi \mathbf{n n}^{T}$.

Proof An integration by parts shows that

$$
\begin{aligned}
\left(a_{\epsilon}-a_{0}\right)\left(u_{0}, p_{\epsilon}\right)= & \int_{\Omega_{\epsilon}} c \nabla u_{0} \cdot \nabla p_{\epsilon}+u_{0} p_{\epsilon} \\
& -\int_{\Omega} c \nabla u_{0} \cdot \nabla p_{\epsilon}+u_{0} p_{\epsilon} \\
= & c \int_{\partial \sigma_{\epsilon}} \partial_{n} u_{0} p_{\epsilon} \\
= & -c \int_{\sigma_{\epsilon}} \nabla u_{0} \cdot \mathbf{n}\left[p_{\epsilon}\right]
\end{aligned}
$$

where $\left[p_{\epsilon}\right]=p_{\epsilon}^{+}-p_{\epsilon}^{-}$, see Figure 1 for the sign convention. Using a double layer potential representation of an approximation of $p_{\epsilon}$ in the free space, it is shown in [1] that an equivalent of the above quantity when $\epsilon \rightarrow 0$ is given by

$$
-c \epsilon^{2} \pi\left(\nabla u_{0} \cdot \mathbf{n}\right)\left(\nabla p_{0} \cdot \mathbf{n}\right)
$$




\section{Connection between elliptic restoration and two-scale edge detection}

\subsection{Different cost functions}

We consider the following three cost functions for which we will derive the associated topological gradients:

$$
\begin{aligned}
& \widetilde{J_{1, \epsilon}}(u)=\int_{\Omega_{\epsilon}} f . u-u^{2}, \\
& \widetilde{J_{2, \epsilon}}(u)=\int_{\Omega_{\epsilon}}-f . u, \\
& \widetilde{J_{3, \epsilon}}(u)=-\int_{\Omega_{\epsilon}} u^{2} .
\end{aligned}
$$

Since $\Omega_{\epsilon}$ coincides with $\Omega$ up to a negligible set, these cost functions do not explicitly depend on $\epsilon$. Henceforth they will be simply denoted by $\widetilde{J_{1}}, \widetilde{J_{2}}, \widetilde{J_{3}}$, respectively. Let us note that

$\widetilde{J_{1}}\left(u_{\epsilon}\right)=\int_{\Omega_{\epsilon}} f \cdot u_{\epsilon}-u_{\epsilon}^{2}=\int_{\Omega_{\epsilon}}-c \Delta u_{\epsilon} \cdot u_{\epsilon}=c \int_{\Omega_{\epsilon}}\left|\nabla u_{\epsilon}\right|^{2}$, which is, up to a constant scaling, the cost function $J_{1, \epsilon}\left(u_{\epsilon}\right)$ introduced in [7]. The minimization of the topological gradient $g_{1}$ associated wih $\widetilde{J_{1}}$ amounts to minimize the $H^{1}$-semi-norm of the perturbed image.

We provide now a connection with another cost function defined in the literature. In [10] the perturbation that is considered is not a crack, and the considered cost function, which depends on two parameters $(\epsilon, \kappa)$, is shown to $\Gamma$-converge to the Mumford-Shah functional for a suitable choice $\kappa=\kappa(\epsilon)$. We adapt the framework of [10] to a crack-shaped perturbation. Therefore we consider the energy cost function:

$$
\begin{aligned}
J_{2, \epsilon}\left(u_{\epsilon}\right) & =\int_{\Omega_{\epsilon}} c\left|\nabla u_{\epsilon}\right|^{2}+\left|u_{\epsilon}-f\right|^{2} \\
& =\int_{\Omega_{\epsilon}}-c \Delta u_{\epsilon} \cdot u_{\epsilon}+\left|u_{\epsilon}-f\right|^{2} \\
& =\int_{\Omega_{\epsilon}} f \cdot u_{\epsilon}-u_{\epsilon}^{2}+\left|u_{\epsilon}-f\right|^{2} \\
& =\int_{\Omega_{\epsilon}} f^{2}-\int_{\Omega_{\epsilon}} f \cdot u_{\epsilon},
\end{aligned}
$$

which is up to an additive constant the cost function $\widetilde{J_{2}}\left(u_{\epsilon}\right)$.

The interpretation of the cost function $\widetilde{J_{3}}$ is the following. The average of $u_{\epsilon}$ does not depend on $\epsilon$ and is the same as the average of $u_{0}$, since

$$
\begin{aligned}
\int_{\Omega_{\epsilon}} u_{\epsilon}-u_{0} & =\int_{\Omega_{\epsilon}}\left(f+c \Delta u_{\epsilon}\right)-\left(f+c \Delta u_{0}\right) \\
& =\int_{\partial \Omega_{\epsilon}} c \partial_{n}\left(u_{\epsilon}-u_{0}\right)=\int_{\partial \sigma_{\epsilon}}-c \partial_{n} u_{0}=0 .
\end{aligned}
$$

This implies that $\widetilde{J_{3, \epsilon}}(u)$ is (up to an additive constant) equal to the opposite of the variance of $u_{\epsilon}$ given by

$$
J_{3, \epsilon}(u)=-\int_{\Omega_{\epsilon}}|u-\bar{u}|^{2}, \quad \bar{u}=\frac{1}{|\Omega|} \int_{\Omega_{\epsilon}} u .
$$

The value of the topological gradient $g_{3}$ associated with $J_{3}$ indicates the decrease of the variance of the image induced by the insertion of an infinitesimal insulating crack. Therefore the minimization of the topological gradient $g_{3}$ amounts to the maximization of the variance of the perturbed image.

\subsection{Topological gradients}

As shown in $[1,2]$, for the cost functions $\widetilde{J_{1, \epsilon}}, \widetilde{J_{2, \epsilon}}$ and $\widetilde{J_{3, \epsilon}}$, hypothesis $\left(H_{1}\right)$ holds with $L$ taken as the Fréchet derivative of the considered functional and $\delta J=0$. Therefore it follows from Theorem 2 that the topological gradients of the cost functions $J_{1}, J_{2}$ and $J_{3}$ are respectively given by:

$$
\begin{aligned}
& g_{1}\left(x_{0}, \mathbf{n}\right)=-\pi c\left(\nabla u_{0}\left(x_{0}\right) \cdot \mathbf{n}\right)\left(\nabla p_{1}\left(x_{0}\right) \cdot \mathbf{n}\right), \\
& g_{2}\left(x_{0}, \mathbf{n}\right)=-\pi c\left(\nabla u_{0}\left(x_{0}\right) \cdot \mathbf{n}\right)\left(\nabla p_{2}\left(x_{0}\right) \cdot \mathbf{n}\right), \\
& g_{3}\left(x_{0}, \mathbf{n}\right)=-\pi c\left(\nabla u_{0}\left(x_{0}\right) \cdot \mathbf{n}\right)\left(\nabla p_{3}\left(x_{0}\right) \cdot \mathbf{n}\right),
\end{aligned}
$$

where the adjoint states solve:

$$
\begin{gathered}
\left\{\begin{array}{l}
-\operatorname{div}\left(c \nabla p_{1}\right)+p_{1}=-f+2 u_{0} \quad \text { in } \Omega, \\
\nabla p_{1} \cdot n=0 \\
p_{2}=u_{0},
\end{array}\right. \\
\begin{cases}-\operatorname{div}\left(c \nabla p_{3}\right)+p_{3}=2 u_{0} & \text { in } \Omega, \\
\nabla p_{3} \cdot n=0 & \text { on } \partial \Omega .\end{cases}
\end{gathered}
$$

\subsection{Link with two-scale edge detection}

Denoting by $u_{00}$ the solution of

$$
\begin{cases}-\operatorname{div}\left(c \nabla u_{00}\right)+u_{00}=u_{0} & \text { in } \Omega \\ \nabla u_{00} \cdot n=0 & \text { on } \partial \Omega\end{cases}
$$

we infer by linearity that $p_{1}=-u_{0}+2 u_{00}$ and $p_{3}=$ $2 u_{00}$.

We will now reformulate these expressions in terms of convolutions. The concept of fundamental solution is used when the domain is the entire plane $\mathbf{R}^{2}$, while the topological asymptotic analysis assumes that the domain $\Omega$ is bounded. However in the case of an image defined in a rectangular domain, the image can be extended by symmetry and periodization to the entire plane $\mathbf{R}^{2}$. We denote by $f^{\sharp}$ the symmetric and periodic extension of $f$ to $\mathbf{R}^{2}$. 
The fundamental solution $\varphi$ of the elliptic restoration equation can be expressed using the modified Bessel function of the second kind $K_{0}$ :

$$
\varphi(x)=\frac{1}{2 \pi} K_{0}(|x| / \sqrt{c}) .
$$

It should be noted that (unlike the fundamental solution for the parabolic heat equation) $\varphi$ presents a singularity at the origin. The kernel $\varphi$ is obtained by radial symmetry from the one-dimensional kernel $\frac{1}{2 \pi} K_{0}\left(\frac{x}{\sqrt{c}}\right)$. The variance of this one-dimensional kernel is

$$
\int_{-\infty}^{+\infty} x^{2} \varphi(x) d x=2 c .
$$

Standard arguments yield

$$
u_{0}=f^{\sharp} \star \varphi .
$$

Likewise it holds

$$
p_{1}=\left(-f^{\sharp}+2 u_{0}\right) \star \varphi=-u_{0}+2 u_{0} \star \varphi,
$$

and

$$
p_{3}=2 u_{0} \star \varphi .
$$

These results are summarized in the following proposition.

Proposition 1 Let us denote by $u_{00}:=f^{\sharp} \star \varphi \star \varphi$ the image $f$ smoothed twice with the kernel $\varphi$. The different topological gradients can be expressed as follows:

$$
\begin{gathered}
g_{1}(., \mathbf{n})=-\pi c\left(\nabla u_{0} \cdot \mathbf{n}\right)\left(\nabla\left(2 u_{00}-u_{0}\right) \cdot \mathbf{n}\right), \\
g_{2}(., \mathbf{n})=-\pi c\left(\nabla u_{0} \cdot \mathbf{n}\right)\left(\nabla u_{0} \cdot \mathbf{n}\right), \\
g_{3}(., \mathbf{n})=-2 \pi c\left(\nabla u_{0} \cdot \mathbf{n}\right)\left(\nabla u_{00} \cdot \mathbf{n}\right) .
\end{gathered}
$$

The interpretation of $g_{2}$ and $g_{3}$ are straightforward: up to the minus sign and a scaling constant,

- $g_{2}$ is the squared norm of the gradient of a smoothed version of the image $f$, projected along the direction $\mathbf{n}$,

- $g_{3}$ is the product of the projections along the direction $\mathbf{n}$ of the gradient of the image $f$ smoothed at two different scales.

The interpretation of $g_{1}$ is less straightforward: it is a combination of the two previous quantities. However for small values of $c$ we can estimate the behavior of $p_{1}=f^{\sharp} \star(2 \varphi \star \varphi-\varphi)$ by a glance at the kernel $\psi=$ $2 \varphi \star \varphi-\varphi$ in the Fourier domain. From

$$
\hat{\varphi}(\xi)=\frac{1}{1+c|\xi|^{2}},
$$

one obtains

$$
\hat{\psi}(\xi)=\frac{2}{\left(1+c|\xi|^{2}\right)^{2}}-\frac{1}{1+c|\xi|^{2}}=\frac{1-c|\xi|^{2}}{1+2 c|\xi|^{2}+c^{2}|\xi|^{4}} .
$$

For a discretized image, the frequencies $\xi$ are bounded above in the Fourier domain, and when $c \rightarrow 0$ the quantity $c|\xi|^{2}$ also tends to 0 . We can then write for small $c$ :

$$
\hat{\psi}(\xi)=\frac{1}{1+3 c|\xi|^{2}+o\left(c|\xi|^{2}\right)},
$$

and the kernel $\psi$ is close to the kernel of the elliptic restoration equation with a constant $3 c$ instead of $c$. For small values of $c$, the quantity $2 u_{00}-u_{0}$ appearing in the expression of the adjoint $p_{1}$ is thus close to a smoothed version of $f$ with the parameter $3 c$.

Since $g_{3}$ combines the gradients of the image at the scales $\sigma=\sqrt{2 c}$ and $\sqrt{2} \sigma$ it is likely to be more robust with respect to noise than $g_{2}$, which only involves the scale $\sigma$. Similarly, for small values of $c$, the quantity $g_{3}$ combines the gradients of the image at the scales $\sigma=$ $\sqrt{2 c}$ and $\sqrt{3} \sigma$ hence the same conclusion is expected to be true. This will be confirmed by our numerical experiments reported in sections 5.2 and 5.4.

\section{Topological gradients for the parabolic heat equation and edge detectors associated with Gaussian kernels}

\subsection{The parabolic heat equation}

We consider the following parabolic heat equation:

$\left\{\begin{array}{l}\partial_{t} u=\Delta u \quad \text { in } \Omega \times[0, T], \\ \nabla u . n=0 \quad \text { on } \partial \Omega \times[0, T], \\ u(t=0)=f .\end{array}\right.$

In the simplified case where $\Omega=\mathbf{R}^{2}$ is the entire space, the heat kernel is a Gaussian with standard deviation $\sqrt{2 t}$. The widely used Canny edge detector [9] is based on the convolution of the image $f$ with a Gaussian of standard deviation $\sigma$, which amounts to consider the solution $u(T)$ of $(10)$ at time $T=\frac{1}{2} \sigma^{2}$. We propose in this section new edge detectors based on Gaussian kernels that generalize the elliptic edge detectors. The elliptic edge detectors were interpreted using two possible viewpoints: topological gradients of different cost functions associated with the perturbation of the elliptic equation on one hand (1) (see section 2), and combinations of gradients of the image $f$ convolved with the fundamental solution $\varphi$ on the other hand (see section 3). We extend these concepts to the parabolic case along the following steps. 
i) We define cost functions associated with the solution of the parabolic equation (10) in a perturbed domain, and calculate the perturbation induced by an infinitesimal insulating crack,

ii) The edge detectors we obtain are expressed in terms of integrals involving the image smoothed at different scales. The calculation of these integrals using a quadrature method provides the numerical versions of these edge detectors. Owing to a priori estimates of the time behavior of the integrands in the regions of interest, we also propose to evaluate these integrals using a single quadrature point, which results in an edge detector involving the image smoothed at two different scales.

The analysis of step i) is presented in sections 4.2 through 4.5 , and step ii) is developed in section 4.6 where we also recall the definition of the Canny edge detector which will be used for comparison purposes.

\subsection{Variational formulation of the heat equation}

We address in this section the continuous-time restoration equation. For a given $T>0$ the state $u_{0}=u_{0}(x, t)$ is the solution of the evolution problem:

$$
\begin{cases}\partial_{t} u_{0}-\Delta u_{0}=0 & \text { in } \Omega \times[0, T] \\ u_{0 \mid t=0}=f & \text { in } \Omega, \\ \nabla u_{0} \cdot n=0 & \text { on } \partial \Omega \times[0, T] .\end{cases}
$$

The image $u_{0}(T)$ is the analysis of the original image $f$ at the spatial scale $\sqrt{2 T}$. We will say that $T$ is the temporal scale of the image $u_{0}(T)$.

For $\epsilon>0$, let again $\Omega_{\epsilon}=\Omega \backslash \sigma_{\epsilon}$, where $\sigma_{\epsilon}$ is the line segment of length $2 \epsilon$ located at the point $x_{0}$ and with unit normal vector $\mathbf{n}$. The perturbed state $u_{\epsilon}$ solves

$$
\begin{cases}\partial_{t} u_{\epsilon}-\Delta u_{\epsilon}=0 & \text { in } \Omega_{\epsilon} \times[0, T], \\ u_{\epsilon \mid t=0}=f & \text { in } \Omega_{\epsilon}, \\ \nabla u_{\epsilon} \cdot n=0 & \text { on } \sigma_{\epsilon} \times[0, T], \\ \nabla u_{\epsilon} \cdot n=0 & \text { on } \partial \Omega \times[0, T] .\end{cases}
$$

We define the following standard function spaces:

$$
\begin{gathered}
Z_{\epsilon}=H^{1}\left(\Omega_{\epsilon}\right), \\
X_{\epsilon, T}=L^{2}\left([0, T], Z_{\epsilon}\right) \cap H^{1}\left([0, T], Z_{\epsilon}^{\prime}\right), \\
X_{\epsilon, T}^{f}=\left\{u \in X_{\epsilon, T} \mid u(., 0)=f\right\} .
\end{gathered}
$$

The variational formulation of problem (12) reads

$$
\left\{\begin{array}{l}
u_{\epsilon} \in X_{\epsilon, T}^{f}, \\
\mathcal{A}_{\epsilon, T}\left(u_{\epsilon}, v\right)=0 \quad \forall v \in X_{\epsilon, T},
\end{array}\right.
$$

with the bilinear form

$$
\mathcal{A}_{\epsilon, T}(u, v)=\int_{0}^{T}\left\langle\partial_{t} u, v\right\rangle+\int_{0}^{T} \int_{\Omega_{\epsilon}} \nabla u . \nabla v .
$$

Above, the notation $\langle.,$.$\rangle stands for the duality pairing$ between $Z_{\epsilon}^{\prime}$ and $Z_{\epsilon}$.

\subsection{Gradient-free cost function}

For mathematical reasons which will become clear later, we first address the parabolic counterpart of $\widetilde{J_{3}}$, considering the time at which the image is analyzed as a variable. Thus, for an arbitrary $\tau \in[0, T]$ we consider the cost function:

$J_{\epsilon}(\tau)=-\frac{1}{2} \int_{\Omega_{\epsilon}}\left|u_{\epsilon}(x, \tau)\right|^{2} d x$.

The scaling constant $1 / 2$ will prove useful for subsequent simplifications. Note that, since $u_{\epsilon} \in \mathcal{C}\left([0, T], L^{2}\left(\Omega_{\epsilon}\right)\right)$ (see $[19,23])$, it follows that $J_{\epsilon} \in \mathcal{C}([0, T])$.

Theorem 3 Assume that $f \in H^{6}(\Omega)$. It holds for all $\tau \in[0, T]:$

$J_{\epsilon}(\tau)-J_{0}(\tau)=\epsilon^{2} G(\tau)+o\left(\epsilon^{2}\right)$

with

$$
G(\tau)=-\int_{0}^{\tau} \nabla u_{0}\left(x_{0}, t\right)^{T} \mathcal{P} \nabla u_{0}\left(x_{0}, 2 \tau-t\right) d t
$$

Above, $u_{0} \in X_{0,2 \tau}^{f}$ is the solution of (11) over the (possibly extended) time interval $[0,2 \tau]$. The polarization matrix is the same as in the elliptic case, i.e. $\mathcal{P}=$ $\pi \mathbf{n n}^{T}$.

Proof We begin by the expansion

$$
\begin{aligned}
J_{\epsilon}(\tau)-J_{0}(\tau)= & -\frac{1}{2} \int_{\Omega}\left(\left|u_{\epsilon}(x, \tau)\right|^{2}-\left|u_{0}(x, \tau)\right|^{2}\right) d x \\
= & -\int_{\Omega} u_{0}(x, \tau)\left(u_{\epsilon}-u_{0}\right)(x, \tau) d x \\
& -\frac{1}{2} \int_{\Omega}\left|\left(u_{\epsilon}-u_{0}\right)(x, \tau)\right|^{2} d x .
\end{aligned}
$$

Then we define the adjoint state $v_{\epsilon, \tau}$ solution of

$$
\left\{\begin{array}{l}
v_{\epsilon, \tau} \in X_{\epsilon, \tau} \\
\mathcal{A}_{\epsilon, \tau}\left(\varphi, v_{\epsilon, \tau}\right)=\int_{\Omega} u_{0}(x, \tau) \varphi(x, \tau) d x \quad \forall \varphi \in X_{\epsilon, \tau}^{0}
\end{array}\right.
$$

In strong form, this reads

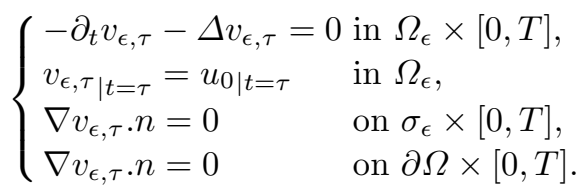

In particular, for $\epsilon=0$, we infer by uniqueness

$v_{0, \tau}(x, t)=u_{0}(x, 2 \tau-t)$. 
Choosing $\varphi=u_{\epsilon}-u_{0}$ in (16) we obtain

$$
\begin{aligned}
J_{\epsilon}(\tau)-J_{0}(\tau)=-\mathcal{A}_{\epsilon, \tau}( & \left.u_{\epsilon}-u_{0}, v_{\epsilon, \tau}\right) \\
& -\frac{1}{2} \int_{\Omega}\left|\left(u_{\epsilon}-u_{0}\right)(x, \tau)\right|^{2} d x .
\end{aligned}
$$

From (13) we have $\mathcal{A}_{\epsilon, \tau}\left(u_{\epsilon}, v_{\epsilon, \tau}\right)=0$. In addition the Green formula yields

$$
\begin{aligned}
\mathcal{A}_{\epsilon, \tau}\left(u_{0}, v_{\epsilon, \tau}\right)= & \int_{0}^{\tau}\left\langle\partial_{t} u_{0}, v_{\epsilon, \tau}\right\rangle+\int_{0}^{\tau} \int_{\Omega_{\epsilon}} \nabla u_{0} . \nabla v_{\epsilon, \tau} \\
= & \int_{0}^{\tau}\left\langle\partial_{t} u_{0}, v_{\epsilon, \tau}\right\rangle+\int_{0}^{\tau} \int_{\Omega_{\epsilon}}-\Delta u_{0} v_{\epsilon, \tau} \\
& +\int_{0}^{\tau} \int_{\sigma_{\epsilon}} \partial_{n} u_{0}\left[v_{\epsilon, \tau}\right] \\
= & \int_{0}^{\tau} \int_{\sigma_{\epsilon}} \partial_{n} u_{0}\left[v_{\epsilon, \tau}\right],
\end{aligned}
$$

where again $\left[v_{\epsilon, \tau}\right]$ stands for the jump of $v_{\epsilon, \tau}$ through $\sigma_{\epsilon}$, see Figure 1. We arrive at

$J_{\epsilon}(\tau)-J_{0}(\tau)=\int_{0}^{\tau} \int_{\sigma_{\epsilon}} \partial_{n} u_{0}\left[v_{\epsilon, \tau}\right]-\frac{1}{2} \int_{\Omega}\left|\left(u_{\epsilon}-u_{0}\right)_{\mid t=\tau}\right|^{2}$.

Combining results from [4] and [1] we obtain the following:

$$
\left\|u_{\epsilon, \tau}-u_{0, \tau}\right\|_{L^{\infty}\left([0, \tau], L^{2}(\Omega)\right)}=o(\epsilon),
$$

$$
\begin{aligned}
\int_{0}^{\tau} & \int_{\sigma_{\epsilon}} \partial_{n} u_{0}\left[v_{\epsilon, \tau}\right] \\
& =-\epsilon^{2} \int_{0}^{\tau} \nabla u_{0}\left(x_{0}, t\right) \mathcal{P} \nabla v_{0, \tau}\left(x_{0}, t\right) d t+o\left(\epsilon^{2}\right)
\end{aligned}
$$

Specifically, counterparts of these results are proven in [4] in the case of an inclusion. The adaptation to the crack case can be achieved using arguments from the static problem addressed in [1]. The reader may also refer to [2] for connections between cracks and inclusions in the topological asymptotic framework. In [4] the conditions $u_{0}, v_{0, \tau} \in L^{2}\left([0, \tau], H^{6}(\Omega)\right) \cap H^{3}\left([0, \tau], L^{2}(\Omega)\right)$ are assumed. Standard parabolic regularity results $[4$, $19,23]$ show that the condition on $u_{0}$ is fulfilled provided that $\Delta^{j} f \in L^{2}(\Omega)$ for every $j \in\{0,1,2,3\}$. The relation (18) shows that $v_{0, \tau}$ enjoys the same regularity (in fact it has even more since $2 \tau-t$ is always positive). Combining (19), (20), (21) and (18) leads to the claimed result.
4.4 Gradient-based cost function

We now turn to the counterpart of $\widetilde{J_{1}}$ given by

$\dot{J}_{\epsilon}(\tau)=\int_{\Omega_{\epsilon}}\left|\nabla u_{\epsilon}(x, \tau)\right|^{2} d x$.

Hille-Yosida's theory shows that $u_{\epsilon} \in \mathcal{C}\left((0, T], H^{1}\left(\Omega_{\epsilon}\right)\right)$, whereby $\dot{J}_{\epsilon} \in \mathcal{C}((0, T])$. Our notation is justified by the following identity.

Lemma 1 For all $\tau \in(0, T]$ it holds

$$
\dot{J}_{\epsilon}(\tau)=\frac{d}{d \tau} J_{\epsilon}(\tau)
$$

Proof Starting from

$$
\dot{J}_{\epsilon}(\tau)=\frac{d}{d \tau} \int_{0}^{\tau} \int_{\Omega_{\epsilon}}\left|\nabla u_{\epsilon}(x, t)\right|^{2} d x d t
$$

and using the variational formulation (13) we arrive at

$$
\dot{J}_{\epsilon}(\tau)=-\frac{d}{d \tau} \int_{0}^{\tau}\left\langle\partial_{t} u_{\epsilon}, u_{\epsilon}\right\rangle .
$$

Assuming for simplicity that $u_{\epsilon}(., t) \in H^{1}\left([0, T], L^{2}\left(\Omega_{\epsilon}\right)\right)$ (the general case follows by density), the above duality pairing is actually an integral, and

$\dot{J}_{\epsilon}(\tau)=-\frac{d}{d \tau} \int_{0}^{\tau} \int_{\Omega_{\epsilon}} \partial_{t} u_{\epsilon} u_{\epsilon}=-\frac{1}{2} \frac{d}{d \tau} \int_{0}^{\tau} \int_{\Omega_{\epsilon}} \partial_{t}\left(\left|u_{\epsilon}\right|^{2}\right)$.

Fubini's theorem yields

$\dot{J}_{\epsilon}(\tau)=-\frac{1}{2} \frac{d}{d \tau}\left(\int_{\Omega_{\epsilon}}\left|u_{\epsilon}(x, \tau)\right|^{2} d x-\int_{\Omega_{\epsilon}}\left|u_{\epsilon}(x, 0)\right|^{2} d x\right)$,

and the proof is complete.

Theorem 4 Assume that $f \in H^{8}(\Omega)$. It holds for all $\tau \in(0, T]:$

$\dot{J}_{\epsilon}(\tau)-\dot{J}_{0}(\tau)=\epsilon^{2} \dot{G}(\tau)+o\left(\epsilon^{2}\right)$,

with

$$
\begin{aligned}
\dot{G}(\tau)= & -\nabla u_{0}\left(x_{0}, \tau\right)^{T} \mathcal{P} \nabla u_{0}\left(x_{0}, \tau\right) \\
& -2 \int_{0}^{\tau} \nabla u_{0}\left(x_{0}, t\right)^{T} \mathcal{P} \partial_{\tau} \nabla u_{0}\left(x_{0}, 2 \tau-t\right) d t .
\end{aligned}
$$

Proof We denote by $S_{\epsilon}(f)$ the solution $u_{\epsilon}$ of the heat equation (12) with initial condition $f$. This defines an operator $S_{\epsilon} \in \mathcal{L}\left(L^{2}(\Omega), X_{\epsilon, T}\right)$. By uniqueness, (17) implies that, for all $t \in[0, \tau]$,

$$
v_{\epsilon, \tau}(., t)=S_{\epsilon}\left(u_{0}(., \tau)\right)(\tau-t) .
$$

Our assumptions entail that $u_{0} \in \mathcal{C}^{1}\left([0, T], L^{2}(\Omega)\right)$, see $[19,23]$. It follows that the map $\tau \in(0, T) \mapsto v_{\epsilon, \tau} \in$ $X_{\epsilon, T}$ is differentiable, with derivative

$$
\dot{v}_{\epsilon, \tau}(., t)=S_{\epsilon}\left(\partial_{t} u_{0}(., \tau)\right)(\tau-t)+\partial_{t} S_{\epsilon}\left(u_{0}(., \tau)\right)(\tau-t) .
$$


By linearity, one has $S_{\epsilon}\left(\partial_{t} u_{0}(., \tau)\right)=\partial_{t} S_{\epsilon}\left(u_{0}(., \tau)\right)$, hence

$$
\dot{v}_{\epsilon, \tau}(., t)=2 \partial_{t} S_{\epsilon}\left(u_{0}(., \tau)\right)(\tau-t)=-2 \partial_{t} v_{\epsilon, \tau}(., t) .
$$

In other words, $\dot{v}_{\epsilon, \tau}$ is the solution of

$$
\begin{cases}-\partial_{t} \dot{v}_{\epsilon, \tau}-\Delta \dot{v}_{\epsilon, \tau}=0 & \text { in } \Omega_{\epsilon} \times[0, T], \\ \left(\dot{v}_{\epsilon, \tau}\right)_{\mid t=\tau}=2\left(\partial_{t} u_{0}\right)_{\mid t=\tau} & \text { in } \Omega_{\epsilon} \\ \nabla v_{\epsilon, \tau} \cdot n=0 & \text { on } \sigma_{\epsilon} \times[0, T] \\ \nabla v_{\epsilon, \tau} \cdot n=0 & \text { on } \partial \Omega \times[0, T]\end{cases}
$$

Next, in view of Lemma 1 and (19), we obtain

$$
\begin{aligned}
& \dot{J}_{\epsilon}(\tau)-\dot{J}_{0}(\tau)=\int_{\sigma_{\epsilon}}\left(\partial_{n} u_{0}\left[v_{\epsilon, \tau}\right]\right)_{\mid t=\tau} \\
& +\int_{0}^{\tau} \int_{\sigma_{\epsilon}} \partial_{n} u_{0}\left[\dot{v}_{\epsilon, \tau}\right]-\int_{\Omega}\left(\left(u_{\epsilon}-u_{0}\right) \partial_{t}\left(u_{\epsilon}-u_{0}\right)\right)_{\mid t=\tau}
\end{aligned}
$$

Similarly to Theorem 3, we have

$$
\begin{aligned}
\int_{0}^{\tau} & \int_{\sigma_{\epsilon}} \partial_{n} u_{0}\left[\dot{v}_{\epsilon, \tau}\right] \\
& =-\epsilon^{2} \int_{0}^{\tau} \nabla u_{0}\left(x_{0}, \tau\right) \mathcal{P} \nabla \dot{v}_{0, \tau}\left(x_{0}, \tau\right) d t+o\left(\epsilon^{2}\right) .
\end{aligned}
$$

Likewise we have for all $\tau \in[0, T]$ (see again $[4,1]$ )

$$
\int_{\sigma_{\epsilon}}\left(\partial_{n} u_{0}\left[v_{\epsilon, \tau}\right]\right)_{\mid t=\tau}=-\epsilon^{2} \nabla u_{0}\left(x_{0}, \tau\right) \mathcal{P} \nabla v_{0, \tau}\left(x_{0}, \tau\right)+o\left(\epsilon^{2}\right) .
$$

By uniqueness from (24), or directly from (18), we infer

$$
\dot{v}_{0, \tau}(x, t)=2 \partial_{t} u_{0}(x, 2 \tau-t) .
$$

The Cauchy-Schwarz inequality yields

$$
\begin{aligned}
& \left|\int_{\Omega}\left(\left(u_{\epsilon}-u_{0}\right) \partial_{t}\left(u_{\epsilon}-u_{0}\right)\right)_{\mid t=\tau}\right| \leq \\
& \left\|\left(u_{\epsilon}-u_{0}\right)(., \tau)\right\|_{L^{2}(\Omega)}\left\|\left(\partial_{t} u_{\epsilon}-\partial_{t} u_{0}\right)(., \tau)\right\|_{L^{2}(\Omega)} .
\end{aligned}
$$

We have already seen that $\left\|u_{\epsilon}-u_{0}\right\|_{L^{\infty}\left([0, \tau], L^{2}(\Omega)\right)}=$ $o(\epsilon)$. Under the appropriate regularity condition, the same estimate holds for $\left\|\partial_{t} u_{\epsilon}-\partial_{t} u_{0}\right\|_{L^{\infty}\left([0, \tau], L^{2}(\Omega)\right)}$ since $\partial_{t} u_{\epsilon}$ solves the same heat equation as $u_{\epsilon}$ with the initial condition $\partial_{t} u_{\epsilon \mid t=0}=\Delta f$. It follows that

$\left|\int_{\Omega}\left(\left(u_{\epsilon}-u_{0}\right) \partial_{t}\left(u_{\epsilon}-u_{0}\right)\right)_{\mid t=\tau}\right|=o\left(\epsilon^{2}\right)$.

Plugging (26), (27) and (28) in (25) leads to the claimed result.
4.5 Edge indicators associated with the parabolic restoration equation

Using the topological asymptotic analysis of the parabolic heat equation (Theorems 3 and 4), we can derive extensions of the edge indicators $g_{1}$ and $g_{3}$. This involves the following cost functions:

$$
\begin{aligned}
K_{1, \epsilon} & =\int_{\Omega}\left|\nabla u_{\epsilon}(x, T)\right|^{2} d x, \\
K_{3, \epsilon} & =-\int_{\Omega}\left|u_{\epsilon}(x, T)\right|^{2} d x .
\end{aligned}
$$

The minimization of $K_{1}$ amounts to minimizing the $H^{1}$ semi-norm of the restored image. The minimization of $K_{3}$ amounts to maximizing the variance of the restored image, as it is straightforwardly proved that the average of $u_{\epsilon}(., T)$ is equal to the average of $f$.

We assume that $f$ is smooth enough (namely $f \in$ $H^{6}(\Omega)$ or $\left.f \in H^{8}(\Omega)\right)$. It follows from Theorems 3 and 4 that the topological gradients associated with the cost functions $K_{1}$ and $K_{3}$ are respectively given by

$$
\begin{aligned}
& h_{1}\left(x_{0}, \mathbf{n}\right)=-\nabla u_{0}\left(x_{0}, T\right)^{T} \mathcal{P} \nabla u_{0}\left(x_{0}, T\right) \\
& \quad-2 \int_{0}^{T} \nabla u_{0}\left(x_{0}, t\right)^{T} \mathcal{P} \partial_{t} \nabla u_{0}\left(x_{0}, 2 T-t\right) d t,
\end{aligned}
$$

$h_{3}\left(x_{0}, \mathbf{n}\right)=-2 \int_{0}^{T} \nabla u_{0}\left(x_{0}, t\right)^{T} \mathcal{P} \nabla u_{0}\left(x_{0}, 2 T-t\right) d t$,

with $\mathcal{P}=\pi \mathbf{n n}^{T}$.

Note: These results were proved in the case where $f$ is very smooth, which is not the case of interest in practice, since noisy images are not much more regular than $L^{2}$ in general. We however believe that the same results hold true for more general hypotheses on $f$, but the proofs require a thorough regularity analysis which is out of the scope of the present work.

There is no direct counterpart of the cost function $\widetilde{J_{2}}$ in the parabolic framework. However, looking at the expression of $g_{2}$ given in Proposition 1, it is natural to define

$h_{2}\left(x_{0}, \mathbf{n}\right)=-\pi\left|\nabla u_{0}\left(x_{0}, T\right) \cdot \mathbf{n}\right|^{2}$,

whose minimization with respect to $\mathbf{n}$ simply gives

$$
h_{2}\left(x_{0}\right)=-\pi\left|\nabla u_{0}\left(x_{0}, T\right)\right|^{2} .
$$

This is, up to the scaling constant $\pi$, the Canny edge detector, which will serve as a reference for our comparisons. 
We denote by $G_{t}$ the heat kernel at time $t$, i.e., the two-dimensional Gaussian kernel with standard deviation $\sqrt{2 t}$. Defining $f^{\sharp}$ as in section 3.3 , we have $u_{0}(., t)=G_{t} \star f^{\sharp}$ and $\nabla u_{0}(., t)=\nabla G_{t} \star f^{\sharp}$. Using this latter expression in (29), (31) and (30) provides the formulations of $h_{1}, h_{2}$ and $h_{3}$ in terms of convolutions.

\subsection{Approximation schemes for the time integrals}

The numerical evaluation of $h_{1}$ and $h_{3}$ requires the computation of the integrals

$$
\begin{aligned}
& I_{1}(x)=\int_{0}^{T} \nabla u_{0}(x, t) \partial_{t} \nabla u_{0}(x, 2 T-t)^{T} d t \\
& I_{3}(x)=\int_{0}^{T} \nabla u_{0}(x, t) \nabla u_{0}(x, 2 T-t)^{T} d t .
\end{aligned}
$$

This has to be done carefully since, on one hand, $\nabla u_{0}(x, t)$ admits a singularity at $t=0$ as soon as $f$ is not smooth at $x$, and, on the other hand, the evaluation of $\nabla u_{0}$ at too many time steps would lead to a prohibitive computer load. We propose two techniques.

i) The first one relies on the observation that $\nabla u_{0}$ can be explicitly integrated, while the other factor in each integral is smooth. Taking the Fourier transform of (11) leads to

$$
\widehat{u_{0}}(\xi, t)=\widehat{f^{\sharp}}(\xi) e^{-t|\xi|^{2}} .
$$

Setting

$$
\widehat{U_{0}}(\xi, t)=\int_{0}^{t} \widehat{f^{\sharp}}(\xi) e^{-s|\xi|^{2}} d s=\frac{\widehat{f^{\sharp}}(\xi)}{|\xi|^{2}}\left(1-e^{-t|\xi|^{2}}\right),
$$

one obtains by inverse Fourier transform an antiderivative $U_{0}(x,$.$) of u_{0}(x,$.$) . Next we consider a regular sub-$ division $t_{0}, \ldots, t_{n}$ of $[0, T]$ with $t_{0}=0$ and $t_{n}=T$. We approximate $I_{3}(x)$ by

$$
\begin{aligned}
I_{3}(x) \approx \sum_{i=0}^{n-1} \frac{1}{t_{i+1}-t_{i}} & \left(\int_{t_{i}}^{t_{i+1}} \nabla u_{0}(x, t) d t\right) \\
& \times\left(\int_{t_{i}}^{t_{i+1}} \nabla u_{0}(x, 2 T-t)^{T} d t\right) .
\end{aligned}
$$

With the help of the antiderivative $\nabla U_{0}(x,$.$) of \nabla u_{0}(x,$.$) ,$ the above integrals are easily computed. One obtains a numerical approximation of $I_{3}$, and proceed similarly for $I_{1}$.

ii) We will now give a very simple approximation of $I_{3}$, using a single quadrature point thanks to an a priori knowledge of the behavior of the function to be integrated. The same idea would apply to $I_{1}$, but our numerical tests showed that only $I_{3}$ was worth this investigation. If $x$ is close to a jump of $f$, say of height $h$ and normal $\mathbf{n}=(1,0)$, then

$$
\nabla u_{0}(x, t) . \mathbf{n} \approx h\left(G_{t} \star \chi_{\left[x_{1}<0\right]}\right)(x)
$$

with $\chi_{\left[x_{1}<0\right]}(x)=1$ if $x_{1}<0$ and $\chi_{\left[x_{1}<0\right]}(x)=0$ otherwise. For $x_{1}>0$ one finds

$$
\nabla u_{0}(x, t) \cdot \mathbf{n} \approx \frac{-h}{\sqrt{4 \pi t}} \exp \left(-\frac{x_{1}}{4 t}\right)
$$

In particular, if $x$ belongs to the jump set, then $\nabla u_{0}(x, t) \cdot \mathbf{n} \approx$ $\alpha(x) / \sqrt{t}$, for some real number $\alpha(x)$. Plugging this expression into (33) entails

$$
\left[I_{3}(x)\right]_{n n} \approx \frac{\pi}{2} \alpha(x)^{2}
$$

Computing now $\left[I_{3}(x)\right]_{n n}$ by the rectangle rule with evaluation point $t^{*}$ results in

$$
T\left(\nabla u_{0}\left(x, t^{*}\right) \cdot \mathbf{n}\right)\left(\nabla u_{0}\left(x, 2 T-t^{*}\right) \cdot \mathbf{n}\right) \approx \frac{T \alpha(x)^{2}}{\sqrt{t^{*}} \sqrt{2 T-t^{*}}} .
$$

Matching the right hand sides of (34) and (35) leads to

$t^{*}=\beta T, \quad$ with $\beta=1-\sqrt{1-\frac{4}{\pi^{2}}} \approx 0.229$.

One derives a two-scale approximation of $h_{3}(x)$, denoted $h_{4}$, given by

$$
h_{4}(x, \mathbf{n})=-2 T u_{0}(x, \beta T)^{T} \mathcal{P} \nabla u_{0}(x,(2-\beta) T) .
$$

\section{Numerical results}

\subsection{Spatial discretization}

We use quadrangular finite elements with $Q 1$ basis functions for the discretization of the Laplace operator. We denote by $K$ the corresponding stiffness matrix. If $u^{V}$ and $u^{\sharp}$ stand for the vector representation and the symmetric and periodic representation, respectively, of an image $u$, one has

$$
K u^{V}=\left(k \star u^{\sharp}\right)^{V},
$$

where $\star$ is the discrete two-dimensional convolution and $k$ is the appropriate kernel. This convolution is efficiently computed using the fast Fourier transform (FFT) and its inverse. This provides fast methods for solving our elliptic and parabolic restoration equations, and subsequently for computing our edge detectors. Note that, as they involve $\nabla u$, these latters are actually computed at the centroids of the elements. 


\subsection{Elliptic restoration}

We implemented the computation of the different topological gradients $g_{1}, g_{2}$ and $g_{3}$. These were applied to a synthetic image with different levels of noise (see Figure 2), and to real images with and without texture (see Figure 4).

Since we aim at detecting edges, we applied a postprocessing algorithm analogous to the Canny edge detector [9]. More precisely, we applied algorithm 1 below.

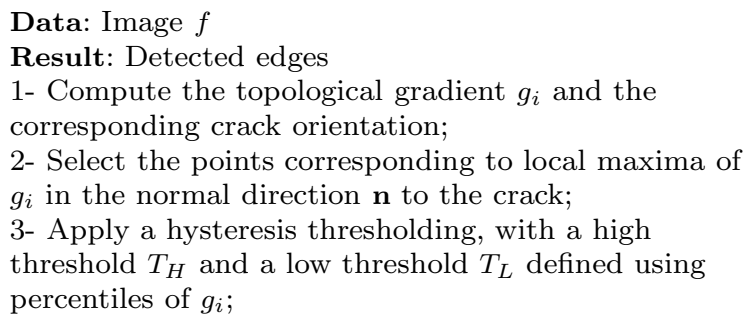

Algorithm 1: Edge detection from $g_{i}$

We observe the following from the synthetic image experiment (Figure 3): at small noise level and small value of $c$, the edge detectors $g_{i}$ are almost similar. At medium and large noise values and larger values of $c$, the detector $g_{1}$ presents artifacts since it doubles the edges. The detector $g_{2}$ presents more noisy values outside the edges, which may not be removed by thresholding. Visual inspection shows that $g_{3}$ provides the best detection of edges.

A possibility to reduce the noise would be to smooth the edge detectors $g_{i}$ before detecting the edges. We did not choose this option in order to highlight the differences between the edge detectors. Moreover, for an enhanced noise reduction outside the edges the method of choice would be to use the parabolic heat equation, see section 5.3.

The topological gradients and detected edges for the cameraman image are presented in Figure 5. The topological gradients and detected edges for Barbara's image are presented in Figure 6. For those images the detectors $g_{1}, g_{2}$ and $g_{3}$ behave relatively similarly. This comes from the fact that our real images were not perturbed by noise. However, differences can be noted for images presenting small details like Barbara. The detector $g_{2}$ finds the edges at the smallest scale. This is coherent with the fact that $g_{2}$ is the squared norm of the gradient of the image smoothed by the kernel $\varphi$, while $g_{3}$ involves the gradients of the image smoothed once and twice by the kernel $\varphi$, and $g_{1}$ roughly involves the gradients of the image smoothed at scale $c$ and $3 c$, since $c$ can be considered as small here. The edge detec-
Table 2 The different values of $T$ used in the parabolic restoration experiments.

\begin{tabular}{lllll}
\hline image & synthetic & synthetic & synthetic & Lena \\
noise & $\eta=0.1$ & $\eta=0.3$ & $\eta=1$ & no noise \\
value of $T$ & $T=0.5$ & $T=2$ & $T=8$ & $T=2$ \\
\hline
\end{tabular}

tors $g_{1}$ and $g_{3}$ are less sensitive to noise, and therefore the small details are smoothed out by these detectors, especially by $g_{1}$.

\subsection{Parabolic restoration}

We implemented the computation of the different topological gradients $h_{1}$ and $h_{3}$, together with the Canny detector $h_{2}$ and the two-scale detector $h_{4}$. The integrals appearing in $h_{1}$ and $h_{3}$ were discretized using the scheme described in section $4.6 \mathrm{i}$ ), where the interval $[0, T]$ was subdivided in 10 subintervals.

The quantities $h_{1}, h_{2}, h_{3}, h_{4}$ were computed for a synthetic image with different levels of noise (see Figure 2 ), and the image of Lena, see Figure 4 right. Different values of $T$ were chosen accordingly to the noise level, see Table 2 . In order to provide results that are visually comparable, we replaced $T$ by $T / 2$ for the detector $h_{2}$, see the discussion in section 5.4. The quantities $h_{i}$ are displayed in Figures 7 and 8. Since these quantities aim at detecting the edges, we applied the same algorithm as in section 5.2 for non-maxima suppression and thresholding.

We observe that the quantity $h_{1}$ is not adapted to edge detection, since it presents a multiple response to edges. The quantities $h_{3}$ and $h_{4}$ are compared with the reference Canny detector $h_{2}$. In Figure 7 the detectors $h_{3}$ and $h_{4}$ appear to be less noisy than $h_{2}$. This comes from the fact that they combine the gradients at different time scales. The detector $h_{4}$ appears to be slightly less noisy than $h_{3}$, because it does not involves gradients at very small time scales. Therefore it seems to be the method of choice in the case of medium and large noise level, providing a good compromise between spatial accuracy and noise reduction.

The detectors were applied to a real image in Figure 8 with $T=2$, which corresponds to a smoothing kernel with standard deviation $\sigma=2$. In the absence of noise, the three detectors $h_{2}, h_{3}$ and $h_{4}$ behave similarly.

As a preliminary conclusion the detector $h_{4}$ proposed here seems to outperform the Canny edge detector in the case of moderate or high noise level. It presents a trade-off between noise reduction and spatial resolution that overtakes the other detectors studied here. This observation is quantitively analyzed in the next section. 

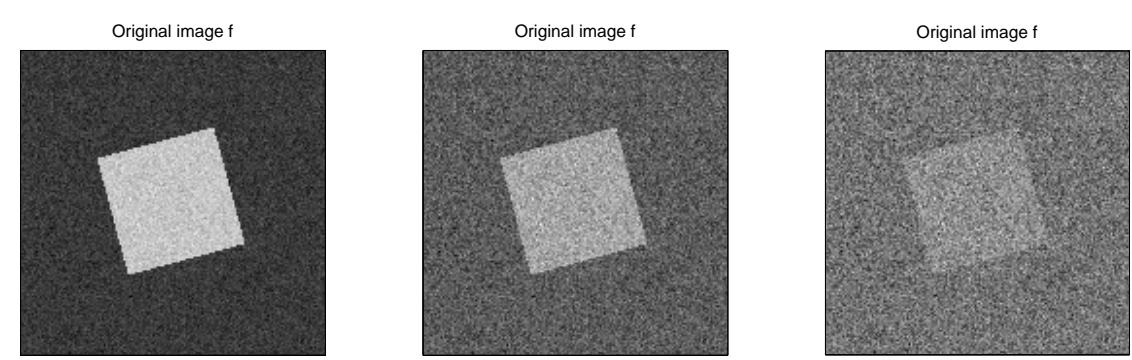

Fig. 2 The synthetic image is the indicator function of a square, perturbed with an additive gaussian random noise of variance $\eta$. We have used the following values (from left to right): $\eta=0.1, \eta=0.3, \eta=1$. The SNR of the images are (from left to right): $11.9 d B, 2.3 d B,-8.1 d B$.
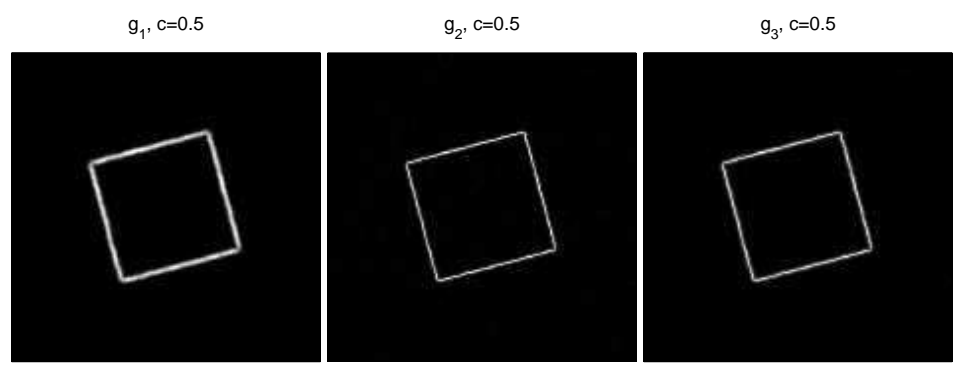

$g_{1}, c=2$

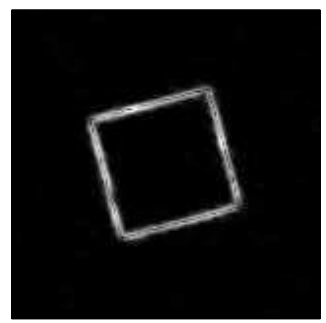

$g_{1}, c=8$

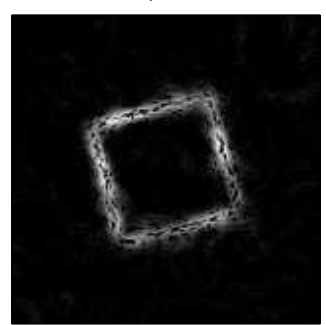

$g_{2}, c=2$

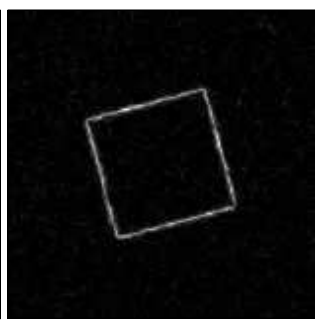

$g_{2}, c=8$

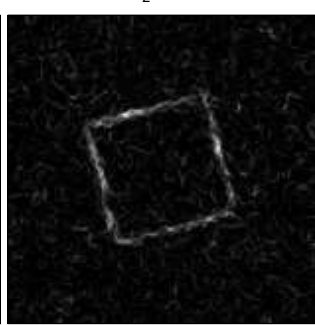

$g_{3}, \mathrm{C}=2$

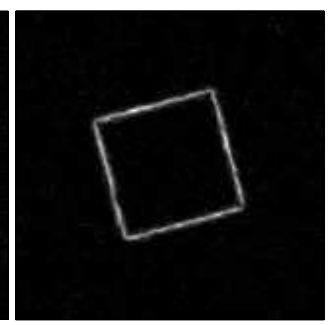

$g_{3}, c=8$

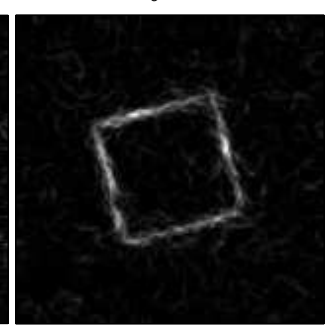

Fig. 3 The topological gradients $g_{1}, g_{2}$ and $g_{3}$ for the low noise case $\eta=0.1$ (top), the medium noise case $\eta=0.3$ (middle), the high noise case $\eta=1$ (bottom). The values of $c$ are respectively $c=0.5, c=2, c=8$ which correspond to a spatial variance of the kernel of resp. $\sigma=1, \sigma=2, \sigma=4$.

\subsection{Quantitative comparison}

All the edge detectors that were presented depend on a smoothing parameter ( $c$ for elliptic detectors, $T$ for parabolic detectors) which should be adjusted depending on the noise level. For a given noise level, different values of the parameter provide more or less smoothed versions of the images which in turn lead to edge detectors having different noise levels and localization accuracy [9]. An oversmoothed filter eliminates fluctuations of the detector due to noise, whereas the localization of the edges becomes less precise. We propose here to evaluate the compromise between noise level and spatial localization of the edge detectors under consideration. 

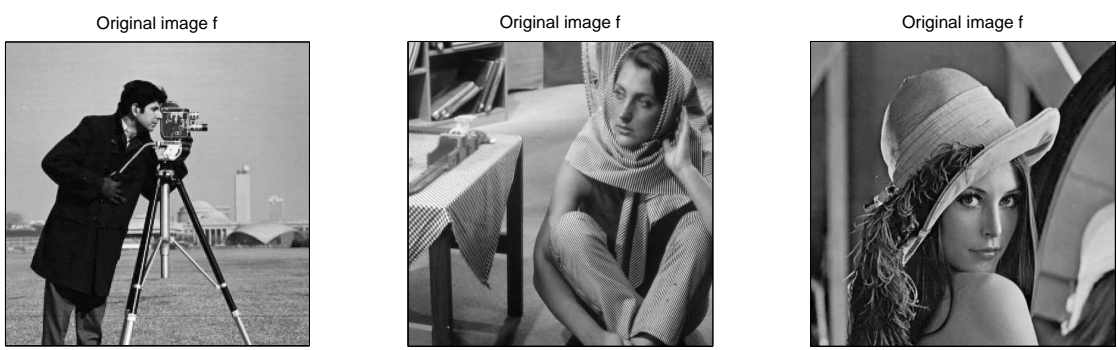

Fig. 4 The cameraman image (left), the Barbara image (center), the Lena image (right).
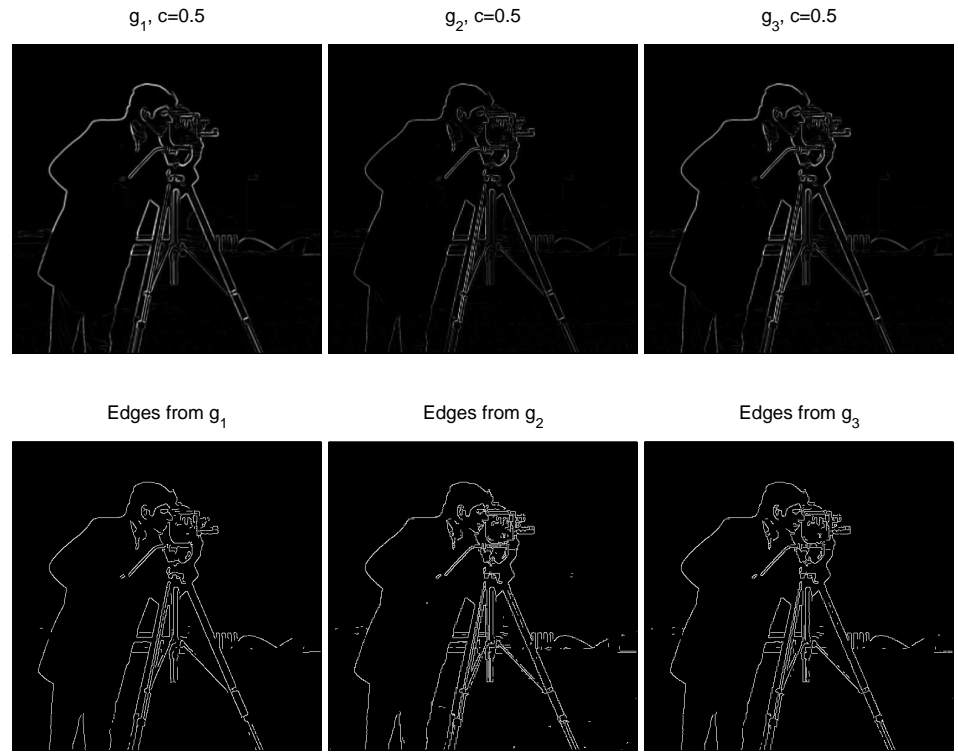

Fig. 5 The topological gradients $g_{1}, g_{2}$ and $g_{3}$ (top) and the detected edges (bottom) for the cameraman image.

We consider a synthetic image with two regions separated by a vertical boundary ( 1 on the left, 0 on the right), degraded by an additive Gaussian noise with variance $\eta$, see Figure 9 (left). The edge detectors are computed for various values of the parameter $(c$ or $T)$, each parameter value allows to estimate a noise level and a spatial resolution which measures the uncertainty in the localization of the edge. These quantities are defined below.

Let us consider an edge detector $d$. This detector is normalized so that its average value along the edge is 1. In practice this normalization is performed by considering the maximum value of the detector along each horizontal line, and averaging these maximum values to obtain a quantity $A$. The detector is normalized so that $A=1$.

Noise: In the homogeneous regions, where no edge is present, an ideal edge detector should provide the value 0 . The quadratic average of $d$ in the homogeneous region gives an estimate of the variance $\sigma^{2}$ of the error on the detector $d$ induced by noise. The noise level is measured in SNR via the formula $-10 \log _{10}\left(\sigma^{2}\right)$.

Spatial resolution: When moving through a segment orthogonal to the edge, the detector presents a peak (normalized to 1). We compute an average of the profiles of this peak over all the horizontal segments in the image, see Figure 9 (right) for an example. Loosely speaking, the more thin is the peak, the more precise is the edge localization. The spatial resolution of the detector $d$ is defined to be the Full Width at Half Maximum (FWHM) of this peak.

For a given detector, when the smoothing parameter ( $c$ or $T$ ) increases, the noise level decreases (the SNR increases) while the localization becomes less accurate (the spatial resolution increases). The trade-off between noise and spatial resolution is thus presented as a curve that gathers the points obtained for different values of the smoothing parameter. 

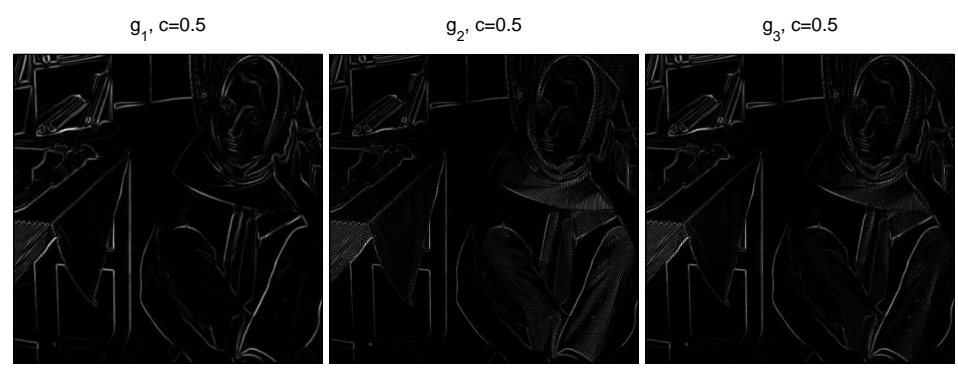

$\mathrm{g}_{1}, \mathrm{c}=0.5$

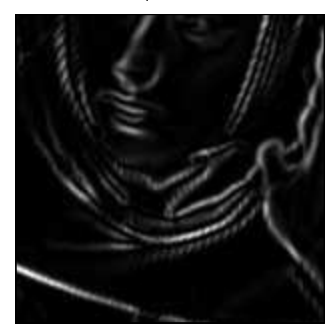

Edges from $g_{1}$

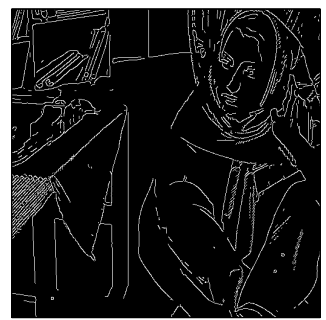

Edges from $g_{1}$

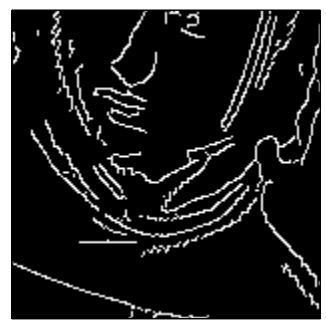

$\mathrm{g}_{2}, \mathrm{c}=0.5$

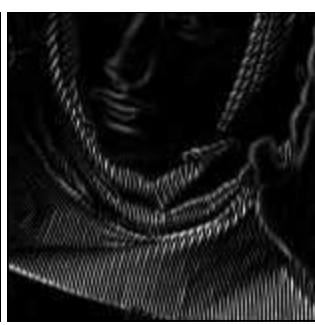

Edges from $g_{2}$

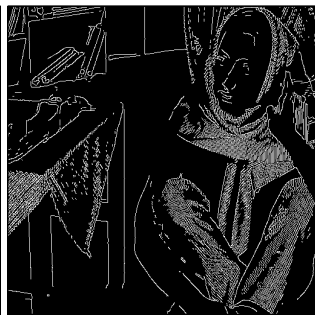

Edges from $\mathrm{g}_{2}$

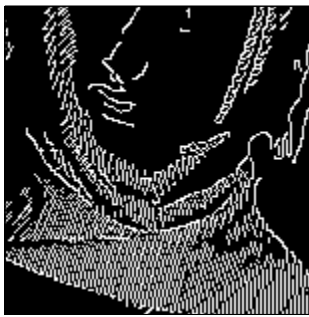

$g_{3}, c=0.5$

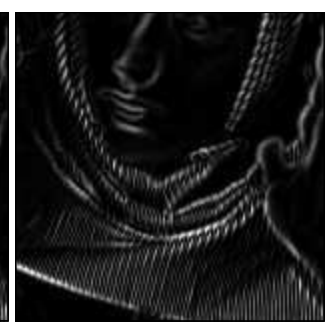

Edges from $\mathrm{g}_{3}$

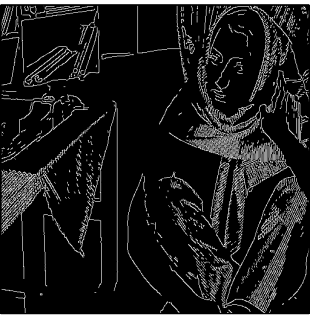

Edges from $\mathrm{g}_{3}$

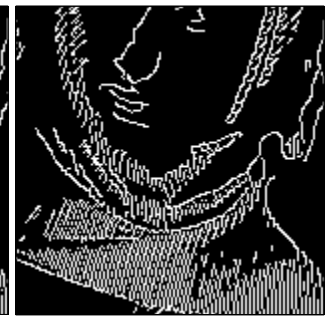

Fig. 6 The topological gradients $g_{1}, g_{2}$ and $g_{3}$ (top rows) and the detected edges (bottom rows) for Barbara's image. We also present zooms of the results in order to show the details close to the scarf. The value $c=0.5$ ensures that the variance of the filter is $\sigma^{2}=2 c=1$

One experiment of the perturbed synthetic image allows to compare the trade-off between noise and spatial resolution for the different detectors $g_{1}, g_{2}, g_{3}, h_{1}, h_{2}, h_{3}$, and $h_{4}$ by comparing the curves associated with the different detectors. The lower is the curve, the better is the detector since for a given noise level, the best detector has the smallest localization error.

The results are presented in Figure 10. We can observe that at low noise level $(\eta=0.1)$ the best performance is obtained by the detectors $h_{4}$ and $g_{1}$ as well as, for small values of the SNR, $h_{1}$. When the noise level in- creases, the best performance is still achieved by $h_{4}$, the performances of $g_{1}$ and $h_{1}$ degrade (due to edge doubling) and the second and third competitors become $h_{3}$ and $g_{3}$. Most of the proposed detectors outperform the Canny edge detector in terms of compromise between noise and spatial resolution.

\section{Conclusions}

We have presented a detailed study of existing edge detection methods based on topological asymptotics. 

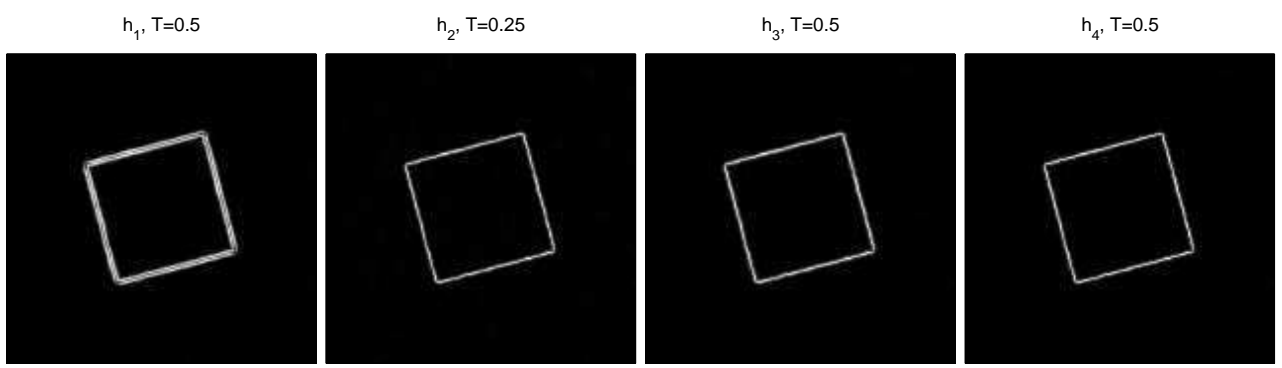

$h_{1}, T=2$

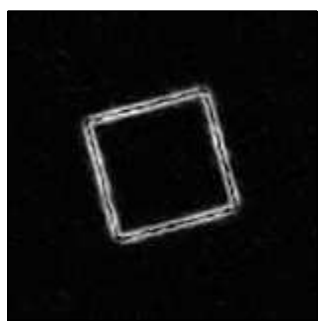

$\mathrm{h}_{1}, \mathrm{~T}=8$

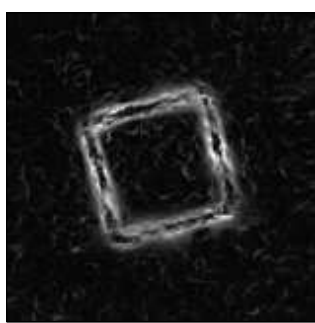

$h_{2}, T=1$

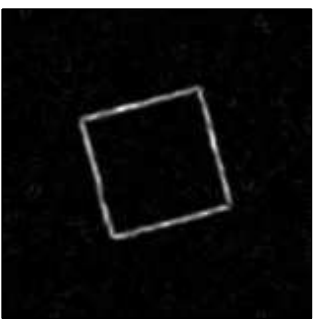

$h_{2}, T=4$

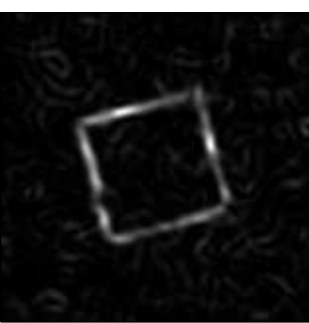

$h_{3}, T=2$

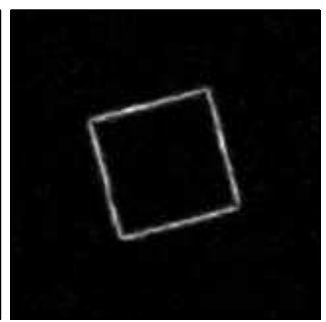

$h_{3}, T=8$

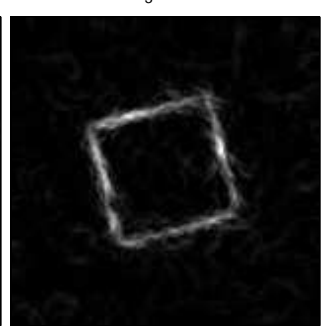

$h_{4}, T=2$

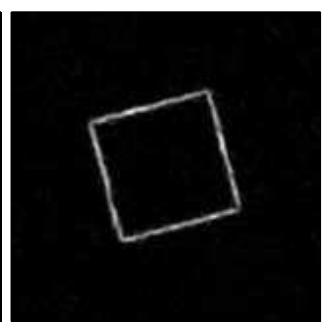

$h_{4}, T=8$

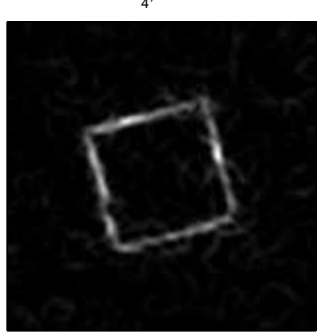

Fig. 7 The parabolic edge detectors $h_{1}, h_{2}, h_{3}$ and $h_{4}$ for the low noise case $\eta=0.1$ (top row), the medium noise case $\eta=0.3$ (middle row), the high noise case $\eta=1$ (bottom row).

We have proposed a new detector associated with the maximization of the variance of the smoothed image using the elliptic restoration equation. This new detector outperforms the two other elliptic detectors in the case of high noise level. We have also extended our approach to the parabolic framework, whose one of the main differences compared to the elliptic setting is that it involves smooth convolution kernels. We have proposed two new edge detectors based on the topological asymptotic analysis of the parabolic heat equation. These detectors extend the elliptic edge detectors in the sense that they are associated with similar cost functions. The integrals appearing in these detectors may be tedious to compute, thus another detector has been proposed, relying on the use of a single wellchosen quadrature point. Numerical experiments show that this latter detector is the most robust. It combines smoothed versions of the image at scales $\sqrt{\beta} \sigma \approx 0.48 \sigma$ and $\sqrt{2-\beta} \sigma \approx 1.33 \sigma$. Therefore it is a two-scale edge detector, where the ratio between the scales stems from our theoretical study.

\section{Acknowledgments}

JF was partly supported by a sabbatical (CRCT) from the National University Council (CNU 26).

\section{References}

1. Amstutz S., Horchani I., Masmoudi M.:, Crack detection by the topological gradient method, Control and Cybernetics 34, 81-101 (2005)

2. Amstutz, S., Dominguez, N.: Topological sensitivity analysis in the context of ultrasonic nondestructive testing, Eng. Ana. Bound. Elem. 32, 936-947 (2008)

3. Amstutz, S.: Sensitivity analysis with respect to a local perturbation of the material property, Asymptotic Analysis 49, 87-108 (2006)

4. Amstutz, S., Takahashi, T., Vexler, B.: Topological sensitivity analysis for time-dependent problems, ESAIM Control Optim. Calc. Var. 14, 427-455 (2008)

5. Auroux, D., Masmoudi, M.: A one-shot inpainting algorithm based on the topological asymptotic analysis, Comp. Appl. Math. 25,1-17 (2006)

6. Auroux, D., Jaafar Belaid, L., Rjaibi, B.: Application of the topological gradient method to color image restoration, SIAM J. Imaging Sci. 3, 153-175 (2010) 

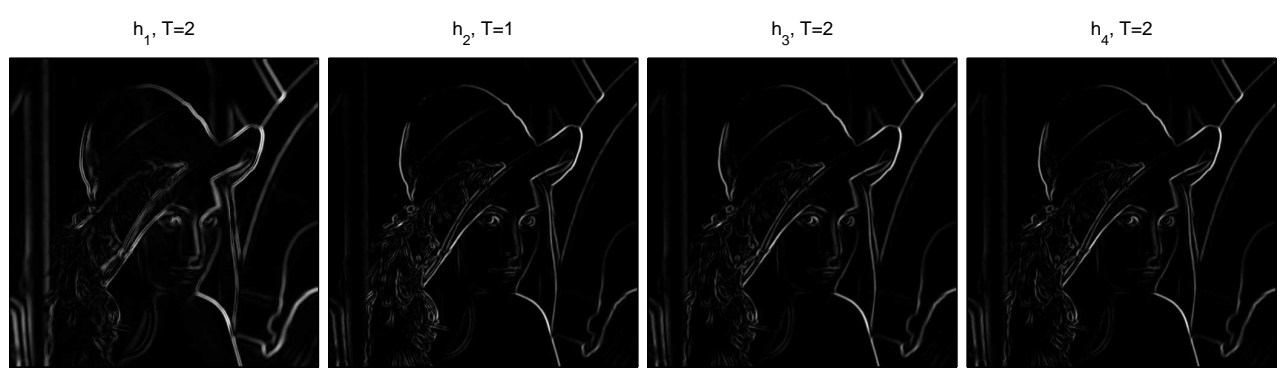

Edges from $\mathrm{h}_{1}$

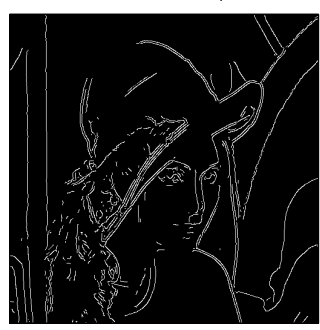

Edges from $\mathrm{h}_{1}$

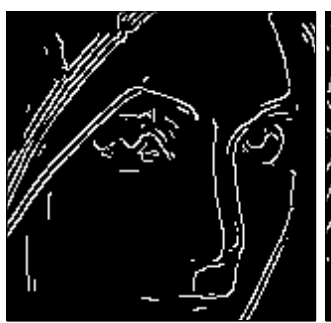

Edges from $\mathrm{h}_{2}$

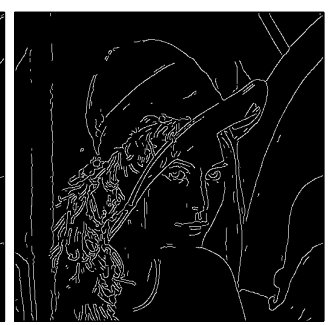

Edges from $\mathrm{h}_{2}$

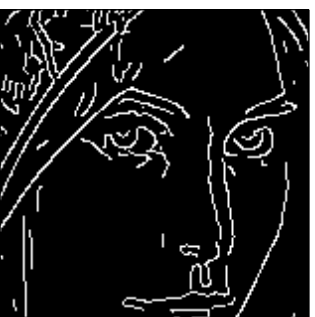

Edges from $\mathrm{h}_{3}$

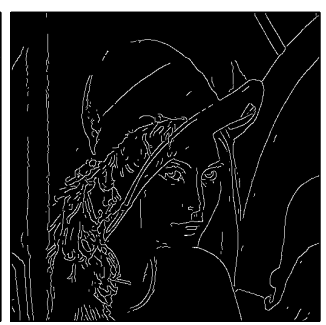

Edges from $\mathrm{h}_{3}$

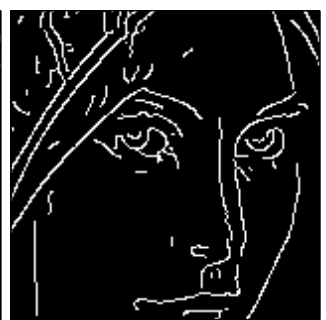

Edges from $h_{4}$

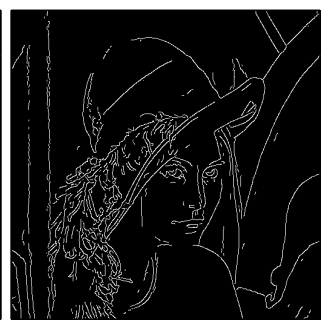

Edges from $\mathrm{h}_{4}$

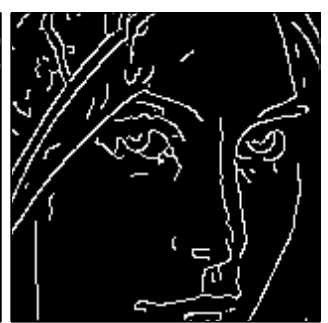

Fig. 8 The edge detectors $h_{1}, h_{2}, h_{3}$ and $h_{3}^{\prime}$ (top row), the detected edges (middle row) and a zoom of the detected edges (bottom row) for the Lena image with $T=2$, which corresponds to a Gaussian kernel with standard deviation $\sigma=2$.
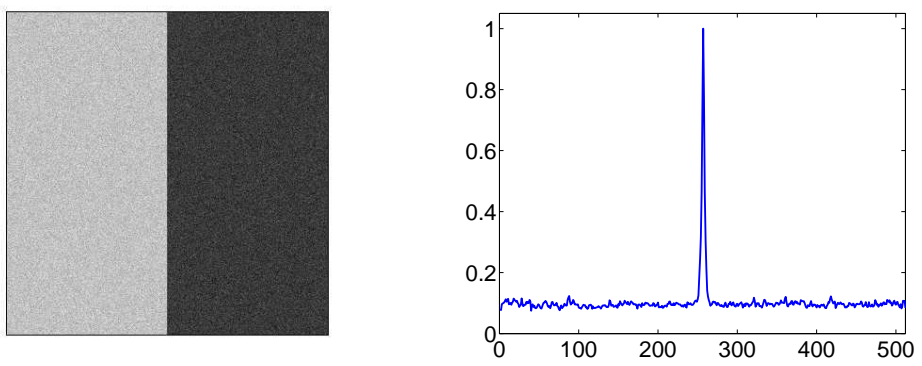

Fig. 9 Left: the synthetic image used for the quantitative comparison, degraded by a Gaussian noise with standard deviation $\eta=0.1$. Right: transverse profile of the edge detector along a direction orthogonal to the edge (averaged over all horizontal lines), the Full Width at Half Maximum (FWHM) of the peak defines the spatial resolution

7. Jaafar-Belaid, L., Jaoua, M., Masmoudi, M., Siala, M.: Image restoration and edge detection by topological asymptotic expansion, C.R.Acad. Sci. Paris, Ser I 342, 313-318 (2006)

8. Beretta, E., Grasmair, M., Muszkieta, M., Scherzer, O., A variational algorithm for the detection of line segments, Inverse Problems and Imaging 8, 389-408 (2014)

9. Canny, J.: A computational approach to edge detection, IEEE Trans. Pattern Analysis and Machine Intelligence 6, 679-698 (1986)
10. Grasmair, M., Muszkieta, M., Scherzer, O.: An approach to the minimization of the Mumford-Shah functional using $\Gamma$-convergence and topological asymptotic expansion, Interf. Free Bound. 15, 141-166 (2013)

11. Koenderink, J.: The structure of images, Biological Cybernetics 50, 363-370 (1984)

12. Larnier S., Fehrenbach J., Masmoudi, M.: The Topological gradient method: from optimal design to image processing, Milan J. Math., 80, 411-441 (2012) 

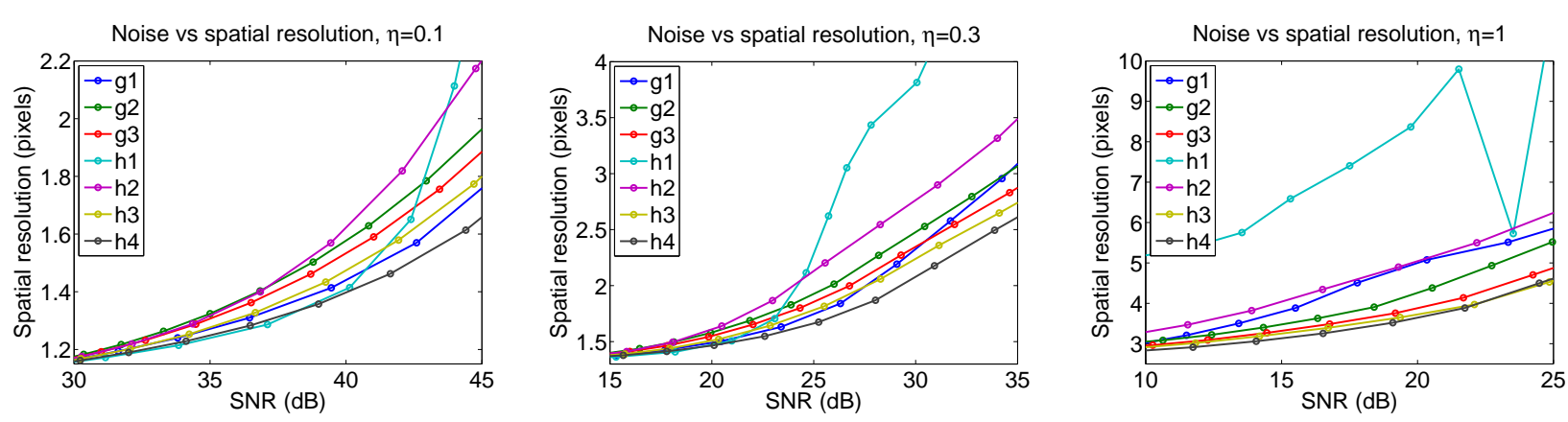

Fig. 10 Trade-off between noise and spatial resolution for the different edge detectors. From left to right: $\eta=0.1, \eta=0.3, \eta=1$.

13. Lindeberg, T. Scale-space theory in computer vision. Springer, 1993.

14. Masmoudi, M. The topological asymptotic, in Computational Methods for Control Applications, H. Kawarada and J. Periaux, eds, GAKUTO Internat. Ser. Math. Sci. Appli. Gakkotosho, Tokyo. (2002)

15. Maz'ia, V., Nazarov, S., Plamenevskij, B.: Asymptotic theory of elliptic boundary value problems in singularly perturbed domains: Vol. 1, Springer (2000)

16. Mumford, D., Shah, J.: Optimal approximations by piecewise smooth functions and associated variational problems, Comm. Pure Appl. Math., 42, 577-685 (1989)

17. Muszkieta, M.: Optimal edge detection by topological asymptotic analysis, Mathematical Models and Methods in Applied Sciences 19, 2127-2143 (2009)

18. Nielsen, M., Deriche, R.: Regularization, scale-space, and edge detection filters, Journal of Mathematical Imaging and Vision 7, 291-307 (1997)

19. Renardy, M., Rogers, R., An introduction to partial differential equations, Springer-Verlag, New York (2004)

20. Schumacher, A.: Topologieoptimierung von Bauteilstrukturen unter Verwendung von Lopchpositionierungkrieterien (Doctoral dissertation, thesis, UniversitatGesamthochschule-Siegen) (1995)

21. Sokolowski, J., Zochowski, A.: Topological derivatives for elliptic problems, Inverse problems 15, 123-134 (1999)

22. Witkin, A.: Scale-space filtering: A new approach to multi-scale description, IEEE International Conference on Acoustics, Speech, and Signal Processing (ICASSP'84) 9 (1984)

23. Wloka, J.: Partial differential equations, Cambridge University Press (1987) 